\title{
L'image qui fait apprendre comme artefact. Théorie instrumentale et enseignement par l'image
}

Jean-Guy Caumeil

\section{QpenEdition}

\section{Journals}

Electronic version

URL: http://journals.openedition.org/trema/3152

DOI: $10.4000 /$ trema.3152

ISSN: 2107-0997

Publisher

Faculté d'Éducation de l'université de Montpellier

Printed version

Date of publication: 1 June 2014

Number of pages: 86 - 99

ISBN: 1167-315X

ISSN: 1167-315X

\section{Electronic reference}

Jean-Guy Caumeil, «L'image qui fait apprendre comme artefact. Théorie instrumentale et enseignement par l'image », Tréma [Online], 41 | 2014, Online since 01 June 2016, connection on 19 April 2019. URL : http://journals.openedition.org/trema/3152 ; DOI : 10.4000/trema.3152

This text was automatically generated on 19 April 2019.

Trema 


\title{
L'image qui fait apprendre comme artefact. Théorie instrumentale et enseignement par l'image
}

\author{
Jean-Guy Caumeil
}

\section{Introduction}

1 Dans cet article nous proposons de considérer l'image comme un artefact. Cette entrée dans l'analyse de l'image fait appel aux théories russes du développement et de l'apprentissage, en particulier à la théorie instrumentale de Vygotski. Pour lui, l'acte instrumental (versus l'acte naturel) a la particularité de s'inscrire dans un milieu caractérisé par la présence de deux types de stimuli : l'objet et l'instrument. L'instrument est à saisir progressivement dans le milieu par le jeu de la conscience. Cette coprésence de l'objet et de l'instrument définit ce que Leontiev (1976) appelle un artefact ${ }^{1}$.

2 Pourquoi et comment une image fait apprendre? À cette question nous répondons par l'hypothèse instrumentale : une image n'est pas qu'une trace, elle porte un « instrument psychologique » (Vygotski, 1930) qui est l'enjeu pédagogique de sa réception. Nous verrons que différents ordres de discours peuvent interférer dans l'acte pédagogique : celui bienveillant, du moins on le suppose, de l'enseignant et celui, quelquefois moins bien veillant du concepteur de l'image.

3 Notre méthodologie consiste à construire et appliquer un modèle de l'artefact à l'analyse sémiotique de l'image.

\section{Le corpus}

4 C'est après la première guerre mondiale que le film pédagogique se développe. Les supports, verre ou papier, étant trop fragiles Pathé invente le Pathéorama. 
Il s'agit de films gélatiniques de 35 millimètres dont on coupe les perforations afin de les rendre utilisables dans des projecteurs conçus par la firme. Les années 1920-1930 voient l'avènement du film 35 millimètres projetable. Le support évoluant et devenant plus accessible (et non inflammable) cette technologie investit l'École. Des « Office du Cinéma Éducateur » (OCE) se créent dans les académies françaises et en 1930 ils sont regroupés au sein d'une Fédération des Offices du Cinéma Éducateur (Caumeil, Charroin, Lignon, Nourrisson, 2010).

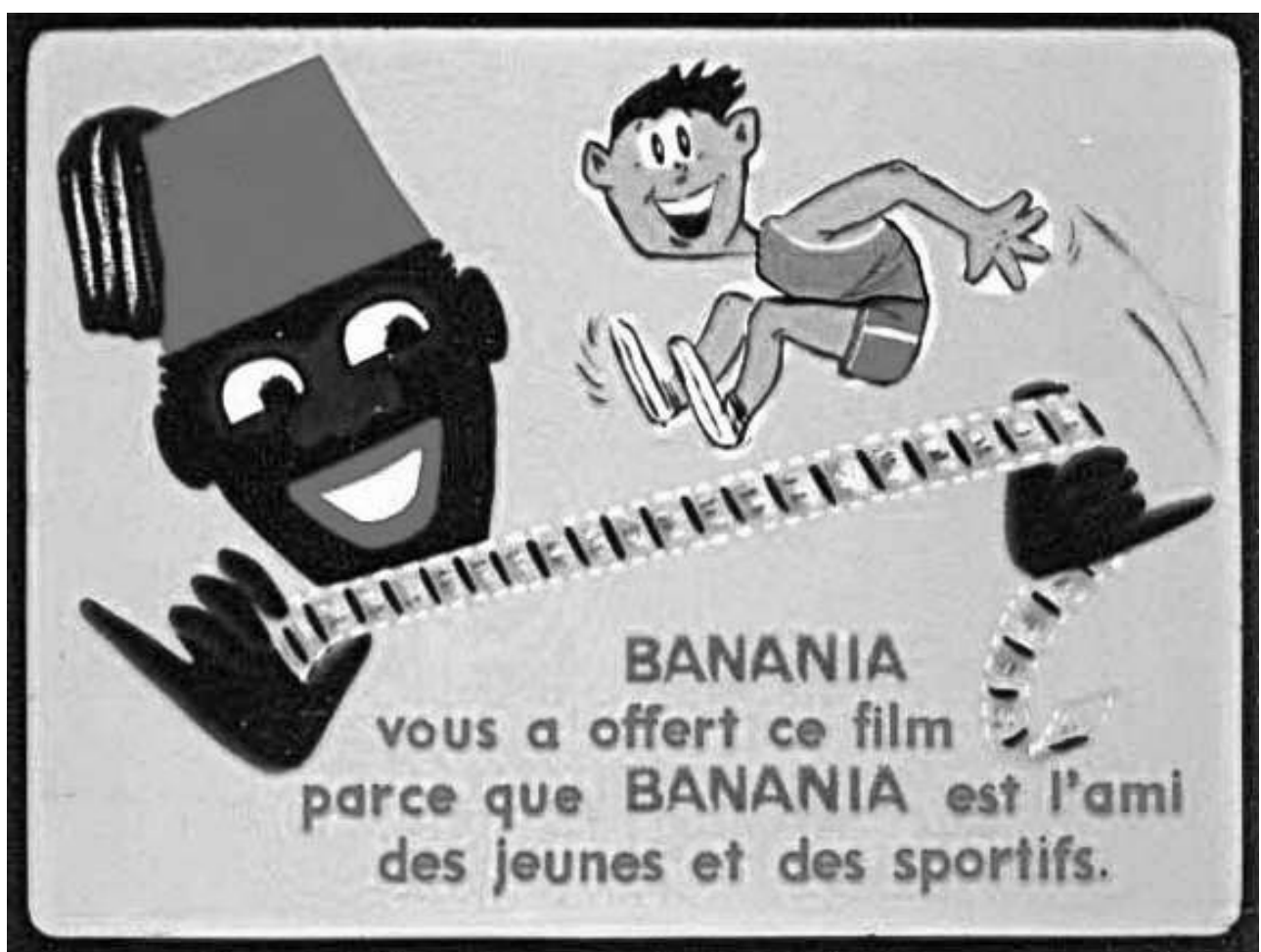

Figure 1 : amorce publicitaire, film le volley-ball, 1960 - 1965

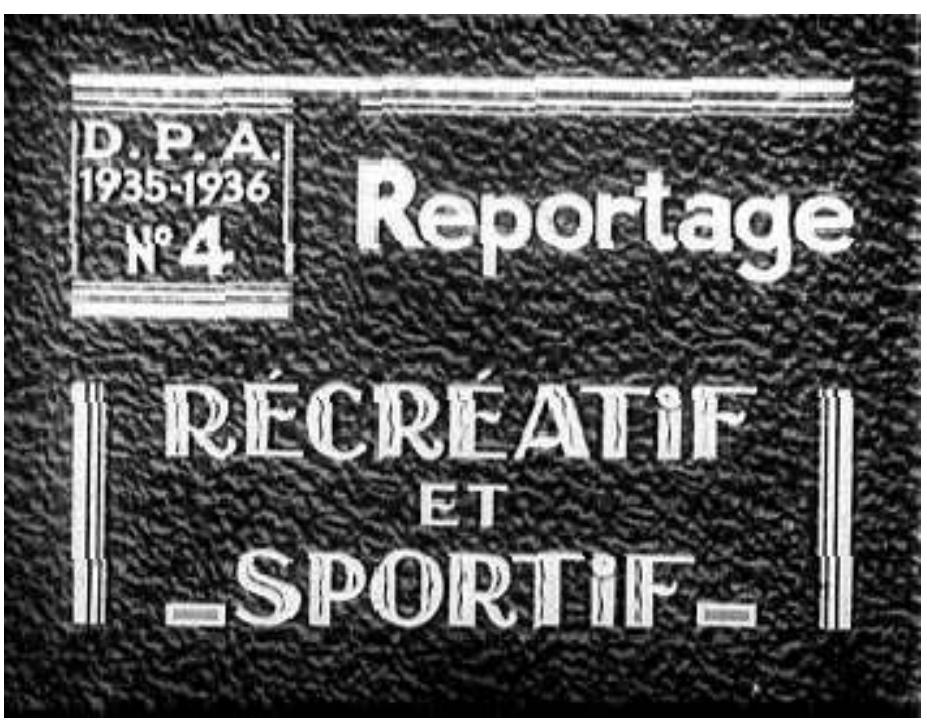

Figure 2 : amorce du film Les jeux Olympiques de 1936

6 Le fonds de films fixes, localisé au site de Saint-Étienne de l'ESPE Lyon1, est constitué du matériel de l'OCE de Saint-Étienne². 


\section{Le sujet}

7 L'utilisation de l'image dans une perspective pédagogique n'est pas récente. C'est Ferdinand Buisson, fraichement nommé Directeur de l'enseignement primaire (1879-1896) qui favorise le recours à l'image. En effet, les lois sur l'école de la troisième république, dites "Lois Ferry", instituent l'obligation scolaire (28 mars 1882); il s'ensuivit dès lors le premier "effet de massification" que notre système scolaire «moderne » subit. Face à ce nouveau défi, Buisson préconise de nouvelles pédagogies, plus innovantes et prenant appuis sur l'image. Reprenant l'ancienne technologie des lanternes magiques, la projection lumineuse à visée d'enseignement de vues sur verre se développe très rapidement à l'école dès la fin du XIXe siècle. Les films fixes prendront le relais dans les années vingt pour faire vivre la pédagogie par l'image jusqu'aux années $1980^{3}$.

8 Pour analyser ces supports nous pouvons utiliser différentes grilles de lecture qui sont le plus souvent héritées du champ de l'analyse filmique. Notre travail a consisté à créer une nouvelle grille à partir de théories qui soient pédagogiques. Comprendre une image pédagogique c'est étudier les signes qu'elle renferme et c'est aussi en percevoir la signification, la sémiose, du point de vue de ce qu'elle est capable de transmettre. Que l'image soit attractive, proliférante, incontournable même, nous n'en doutons pas, qu'elle permette d'apprendre est une toute autre affaire. Et quand bien même la chose est acquise, que nous permettent d'apprendre les images pédagogiques?

\section{Théorie épistémo-anthropologique et paradigme médiationniste}

9 Pour Vygotski, les activités humaines s'expriment et se développent dans deux sortes d'actes. Les actes naturels et les actes instrumentaux. Les connaissances produites dans un acte naturel sont portées par l'objet et proviennent des informations perceptives. Vygotski qualifie ces connaissances de spontanées. Ces connaissances sont multiples, utiles, mais non suffisantes pour expliquer complètement certains faits ou résoudre certains problèmes complexes. Pour cela l'homme doit inventer des explications et des solutions qui dépassent le traitement des informations inscrites dans les données immédiates de l'expérience sensible. D’emblée nous voyons la possibilité de passer du niveau général de la théorie à son application à l'image, et par extension à l'» éducation à l'image ». En tant qu'objet créé par l'homme, l'image est un produit culturel que l'on peut recevoir au niveau sensible (empirique, émotionnel, premier...) mais que l'on peut aussi analyser puisque, justement, c'est un construit culturel. Le discours sur l'image représente alors le savoir théorique visé par l'éducation à l'image.

Ces savoirs sont des cadres d'interprétations et d'actions qui sont des adaptations artificielles produites par l'homme hic et nunc. Savoirs et interprétation de l'image sont donc les enjeux de l'éducation par et à l'image. En termes vygotskiens on parle d'» enjeux symboliques ». Ces adaptations artificielles qui sont des signes, des concepts, des normes ou des techniques, portent des instruments psychiques supérieurs (mots, symboles, usages, savoir-faire, heuristiques, règles, lois, etc.) qui sont les médiateurs sémiotiques de la vie psychique de l'homme. De ce point de vue, Vygotski pose deux types de médiations. 
La médiation des instruments psychologiques supérieurs qui permet à l'homme de construire un rapport au monde historiquement situé et ancré sur les savoirs dans sa culture; et la médiation incarnée par le maitre qui tire le développement psychique de l'élève dans une zone du plus proche développement où il réalisera avec l'aide du maitre ce qu'il ne peut pas encore faire seul. En appliquant les concepts de la théorie instrumentale nous pouvons dire que l'image pédagogique participe de deux ordres de médiation : elle porte des instruments psychologiques qui sont les enjeux symboliques de la situation d'enseignement, c'est la médiation instrumentale, et elle participe comme support du discours du maitre à la médiation incarnée.

11 La théorie instrumentale pose de façon forte l'impact des savoirs scientifiques sur la construction de l'homme au-delà de l'élève. Caumeil $(2000,2006)$ appelle ce phénomène la dimension épistémo-anthropologique de l'éducation scolaire.

L'acte instrumental (versus l'acte naturel) a la particularité de s'inscrire dans un milieu caractérisé par la présence de deux types de stimuli : l'objet et l'instrument. L'instrument est à saisir progressivement dans le milieu par le double jeu de la conscience, conscientisant (rendant objectif le monde) et conscientisante (portant sur le monde une réflexion et un discours).

Cette coprésence de l'objet et de l'instrument définit ce que Leontiev (1976) appelle un artefact. Qu'est-ce qu'un artefact? (Nous pourrions dire pareillement, qu'est-ce qu'une image?

Pour Wartofsky $(1979,2004)$ les artefacts sont « des objectivations des besoins et des intentions humaines; c'est-à-dire comme déjà investis d'un contenu cognitif et affectif. L'outil est appréhendé à la fois dans son utilisation, et dans sa production, dans une perspective instrumentale, comme quelque chose destiné à être fait pour et employé selon un certain but ».

Appliquée à l'image, en général, cette définition fait ressortir le fait qu'une image n'est jamais neutre, elle porte un contenu cognitif spécifique à la nature même de l'image (de l'image de vacances, à l'image publicitaire, à l'image de propagande, en passant par l'image scientifique) de même qu'un contenu affectif qui peut représenter une très forte charge émotionnelle suivant le sujet représenté. À cela s'ajoute le fait que l'image fixe, entendons par là une image dont la visée est pédagogique, est destinée à "être faite pour » et qu'on l'utilise "pour atteindre un but». Analyser une image fixe à visée éducative, consiste à combiner les quatre dimensions. On comprend qu'une image a un très fort impact sur celui qui la construit et sur celui qui la reçoit. De plus elle porte une inclinaison à la manipulation, plus ou moins consentie, de l'autre (fonction conative).

L'artefact est un ensemble complexe qui dépasse par exemple un «contenu d'enseignement ». L'artefact est composé :

- d'une étiquette conceptuelle, nom ou discours ;

- d'un (ou des) instrument psychologique qui renvoie à une adaptation artificielle (outil, technique, forme langagière, opération, etc.) conçue par l'homme dans un temps et dans une culture spécifiques pour répondre aux problèmes qu'il rencontre dans son rapport au monde. Dans ce sens, l'artefact (l'objet plus l'instrument), ne peut se concevoir que dans sa dimension historico-culturelle et selon sa portée artefactuelle. La portée artefactuelle de l'objet s'apprécie au regard de l'efficience des instruments psychiques portés par l'artefact ;

- enfin il revêt une dimension affective et conative (une propension à agir ou à percevoir selon une direction précise). 
17 Nous voyons que l'expertise du maitre tient à la maitrise des instruments psychologiques portés par les supports pédagogiques qu'il utilise. En effet l'image, et donc par voie de conséquence l'image pédagogique, par son apparente facilité de lecture, son approche attractive et directe, peut prendre en défaut cette expertise. Nous avons constaté en regardant certains films fixes que des connaissances plus ou moins justes ou des idéologies étaient véhiculées par les images pédagogiques de façon insidieuse. Poser l'analyse d'image par l'entrée des instruments psychologiques permet de repérer ce qui serait plus ou moins douteux dans le contenu formel et informel de l'image. Cette expertise permettrait peut-être d'éviter que des pseudo-savoirs ou des idéologies soient propagés à l'insu de l'enseignant.

\section{Éléments d'une sémiologie de l'image d'enseignement}

Roland Barthes pose la question : peut-on appliquer à l'image les concepts et les méthodes de l'analyse littéraire ? Pour lui ${ }^{4}$, la sémiologie est une méthode d'analyse à caractère extensif dans la mesure où elle s'applique à tous les signes ${ }^{5}$.

Qu'est-ce qu'un signe? L'image est-elle un signe ? Tout est-il signe?

Constituer les éléments d'une sémiologie de l'image d'enseignement consiste donc pour nous à emprunter la voie tracée par Roland Barthes, mais nous ne nous appuierons que sur une partie des concepts qu'il propose. En effet, le cadre restreint de cette communication ne nous permet pas de développer le concept de Langue/Parole, ni d'aborder la question de fond qui divise Saussure et Peirce sur la définition du signe. Postulons seulement que le concept de Langue s'applique à tous les actes de communication y compris ceux dont la substance n'est pas verbale ${ }^{6}$. En ce sens, le langage photographique utilise une langue dont $\mathrm{Hjelmslev}^{7}$ nous dit qu'elle a :

- un schéma : les règles de compositions, physico-chimiques mais aussi artistiques, de la photographie : les couleurs, le contraste, l'éclairage ;

- une norme : le cadre plan où la réalité est re-présentée - dimension, échelle, substance - ;

- un usage : la photographie comme ensemble d'habitudes d'une société donnée - le contexte, le cadrage -. 

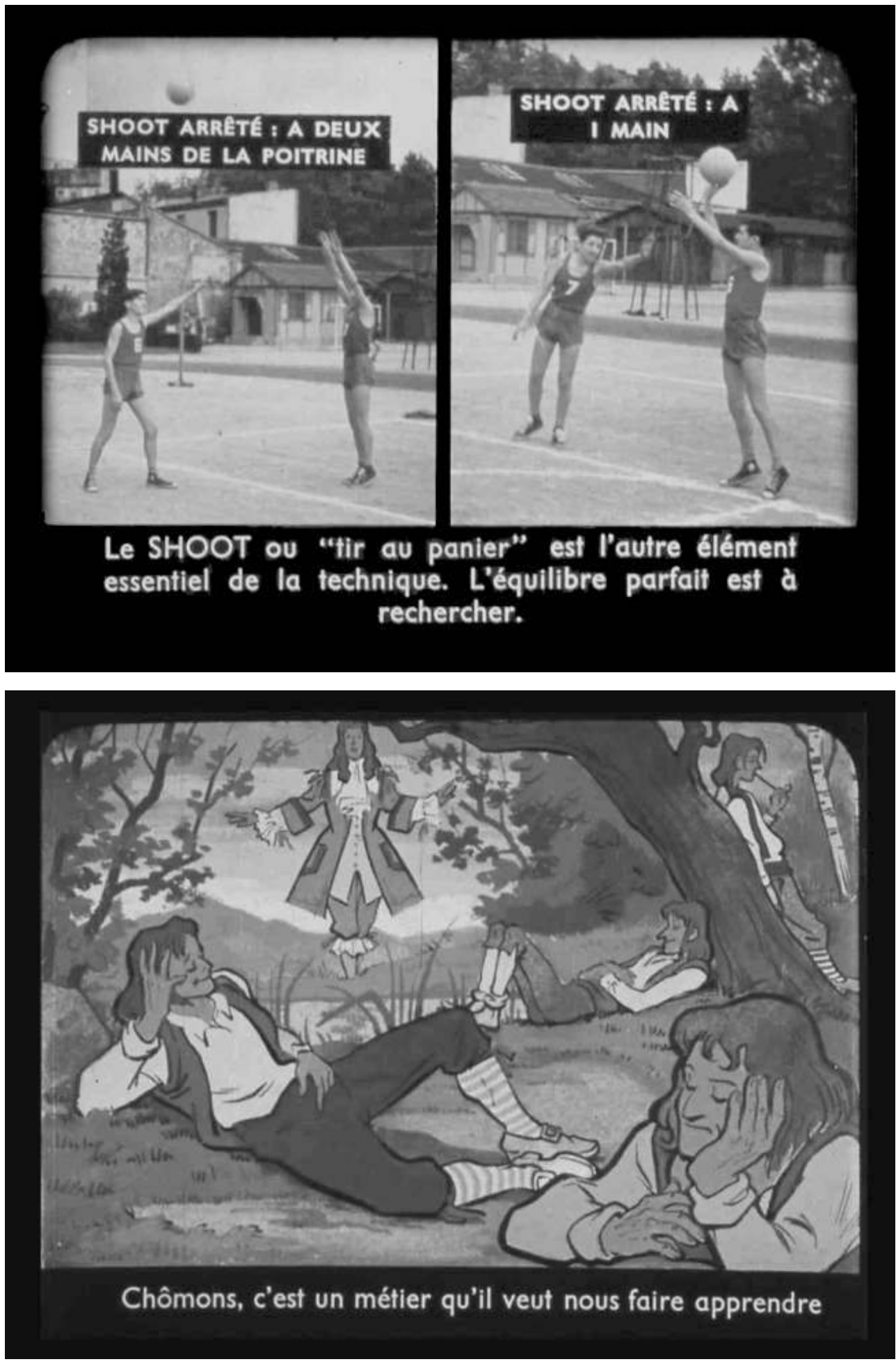

Figure 3 et 4 : Différentes dimensions (norme, technique, concept, outil) de l'analyse sémiotique de l'image d'enseignement

Ces trois constituants du langage iconique cristallisent l'ensemble (non exhaustif puisque le sujet de l'image porte ses propres instruments psychologiques) des instruments psychologiques supérieurs portés par l'artefact image pédagogique (règles, normes, techniques de prises de vue, concepts de composition d'images, etc.). Par ailleurs, les rapports entre «la parole» - ce que l'on veut dire - et le schéma, la norme et l'usage 
enrichissent la langue - le moyen de le dire - en jouant sur les combinatoires. De même, le contraste d'une photographie donnera plus ou moins de dramatisation à la scène représentée - c'est la combinatoire parole-schéma. La dimension, souvent plus petite, de la photographie oblige l'observateur à recourir à la reconnaissance de la scène pour comprendre et interpréter - c'est la combinatoire parole-norme. La photographie des mariés nécessite la prise de vue en pied - c'est la combinatoire parole et usage. Ces combinatoires d'instruments élémentaires constituent à leur tour des instruments psychologiques.

\section{Image et pédagogie : un rapport complexe}

21 Les films fixes, ou les images fixes, de sports ou d'éducation physique sont des photographies, principalement, mais aussi des dessins, des gravures voire des schémas ou des diagrammes. Ils présentent une activité physique au sens large du terme puisqu'on trouve des films d'actualité, concernant les Jeux Olympiques ou la vie de Jacques Anquetil, aussi bien que des films de sports concernant la technique motrice ou des films ventant les vertus du camping ou des colonies de plein air. La recherche sémiotique nous alerte sur le fait que l'image, principalement la photographie, n'est pas le référent mais la trace qu'il laisse sur une surface sensible grâce à l'action de la lumière. Il serait plus conforme, comme certains auteurs le proposent, de qualifier ces représentations du réel d'» icônes » ou d' "analogons ». Ces dénominations ont le mérite de rappeler que l'objet entretient avec le réel un degré, plus ou moins important, d'analogie ${ }^{8}$. Pour M. Martin (1982) «[...] l'icône photographique passe par différentes transformations avant que le récepteur reconnaisse l'image du référent. [...]. Ces codes, que nous appelons pseudo-naturels parce qu'ils paraissent aller de soi, concernent, entre autres : Les dimensions [...]. L'échelle [...].

La substance [...]. Les couleurs [...]. L'éclairage [...]. Le contexte [...]. Le cadrage [...]. Ainsi, contrairement à ce qu'une approche immédiate et naïve peut inférer, l'image, même photographique, est loin d'équivaloir à la réalité ».

22 Les films fixes font partie des outils de communication que les méthodes modernes, actives, ont mises en œuvre à partir des années $1920^{\circ}$. Parce que, durant de nombreux siècles, les pédagogues ont presque exclusivement fondé leur action sur leur parole et leur exemple. Certes, des images pieuses, ou édifiantes, circulaient largement par l'intermédiaire des colporteurs, d'assez nombreux manuels didactiques illustrés existaient aussi, pensons par exemple L'orbissensualium Pictus de Coménius (1656), mais l'expression première de l'enseignement était bien l'exposition magistrale du savoir.

Qu'est-ce qui a conduit les pédagogues à utiliser des surfaces d'inscription pour enrichir leurs enseignements? L'étude du fonds permet de constater que les films fixes sont construits sur un même modèle, celui de la leçon type des années cinquante, modèle dominant à cette période et encore très présent dans les représentations communes de l'enseignement. On retrouve la référence à la leçon type citée in extenso dans les notices accompagnant les films. Les contenus sont présentés de façon progressive, ils sont regroupés en fonction de thèmes d'étude ou de chapitres et sont accompagnés des erreurs à ne pas commettre. Mais dans le même temps, ils recouvrent des spécificités selon qu'ils traitent de l'éducation physique ou des sports, de l'histoire ou du français, des sciences, etc. Cette remarque permet de comprendre que la portée artefactuelle des images pédagogiques dépasse les seuls contenus disciplinaires. En l'occurrence, la leçon type est 
véhiculée comme une norme scolaire qui s'incorpore au fil des séances et des disciplines, mais qui structure aussi le montage des films fixes.

Par ailleurs, les films fixes d'éducation physique sont très souvent " sponsorisés " par des marques (agroalimentaires, pétrolières, industrielles, etc.), parfois par des chambres syndicales ou des collectivités territoriales.

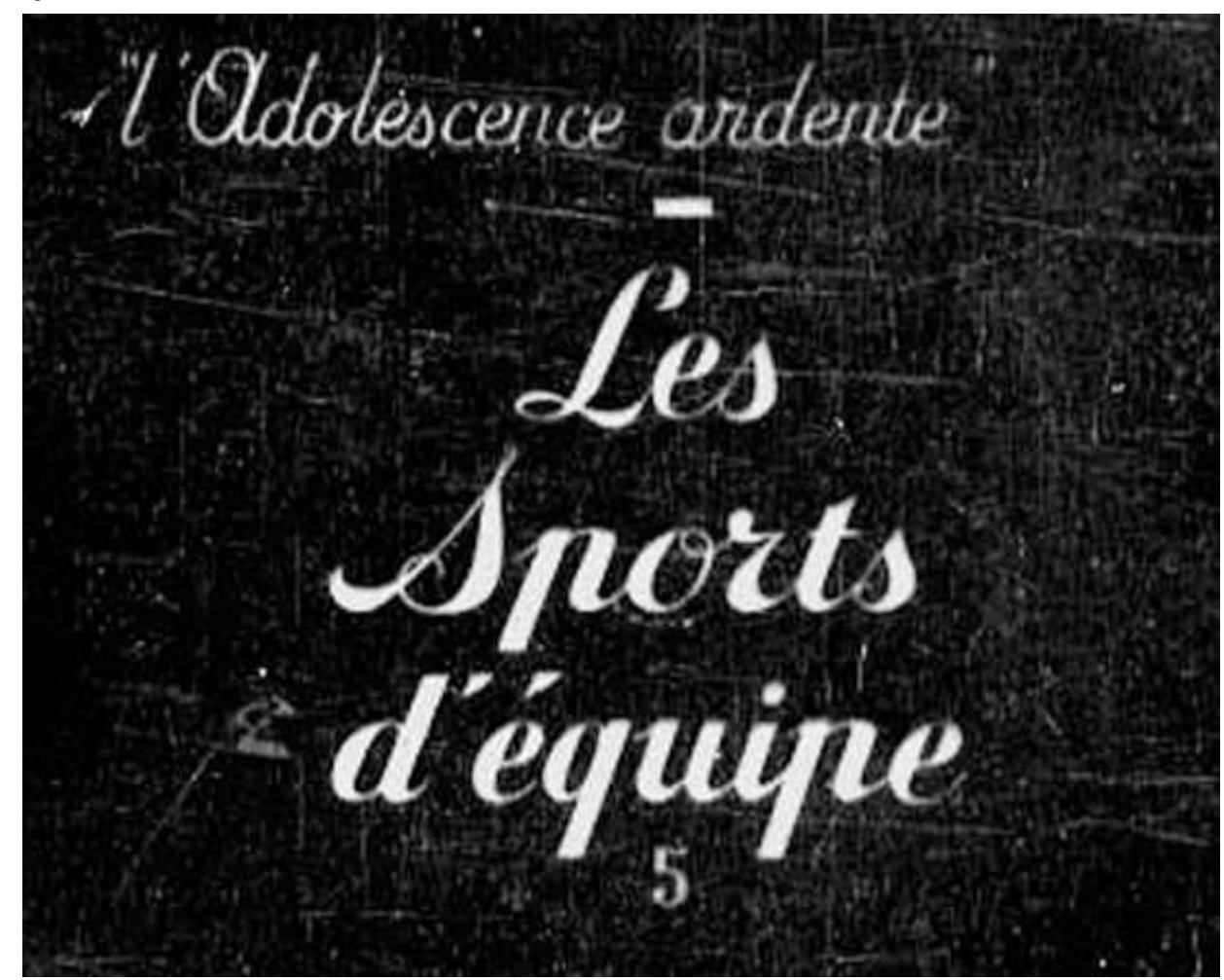

Figure 5 : Film l'athlétisme $7^{\text {re }}$ partie

De mystérieuses organisations - l'Adolescence Ardente par exemple, dont on ne retrouve aucune trace de nos jours, parrainent des films que les maisons d'édition distribuent.

L'École, via les films fixes d'éducation physique, devient le terrain de prédilection d'une publicité bon marché pour les firmes. Cette publicité prend largement appui sur les aspects hygiéniques et sanitaires de la pratique physique.

Il se développe, surtout dans les années quarante, un endoctrinement, voire une propagande, qui n'est pas toujours subtile comme nous le verrons par la suite. Là encore, considérer l'image pédagogique comme un artefact nous permet de séparer ce qui, dans le support pédagogique, appartient à l'école de ce qui devrait lui rester étranger. 


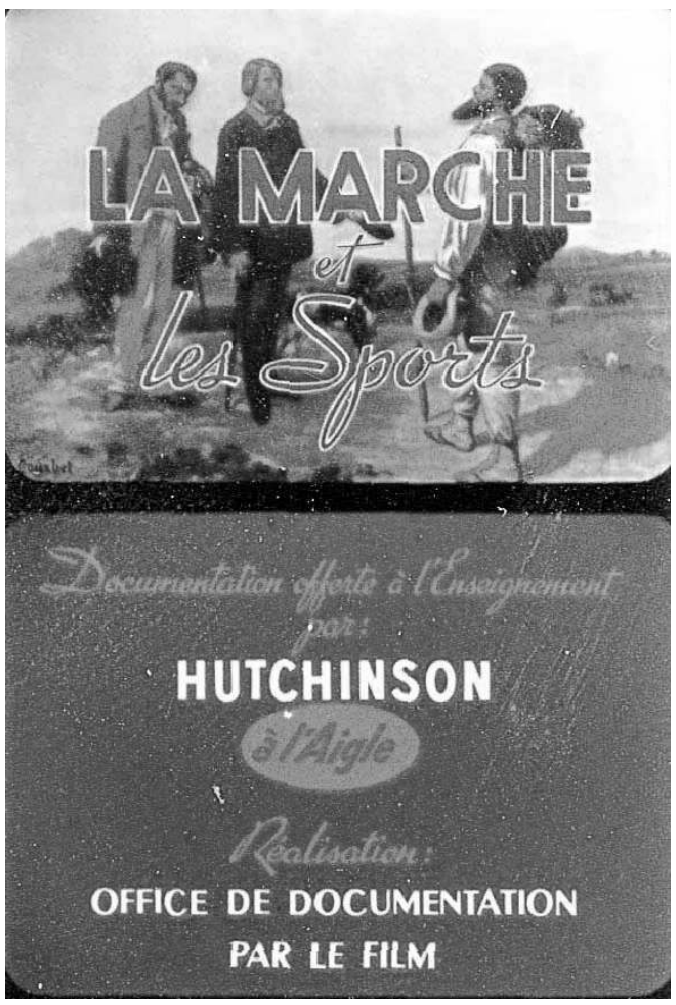

Figure 6 : amorce publicitaire, film la marche, 1960-1965

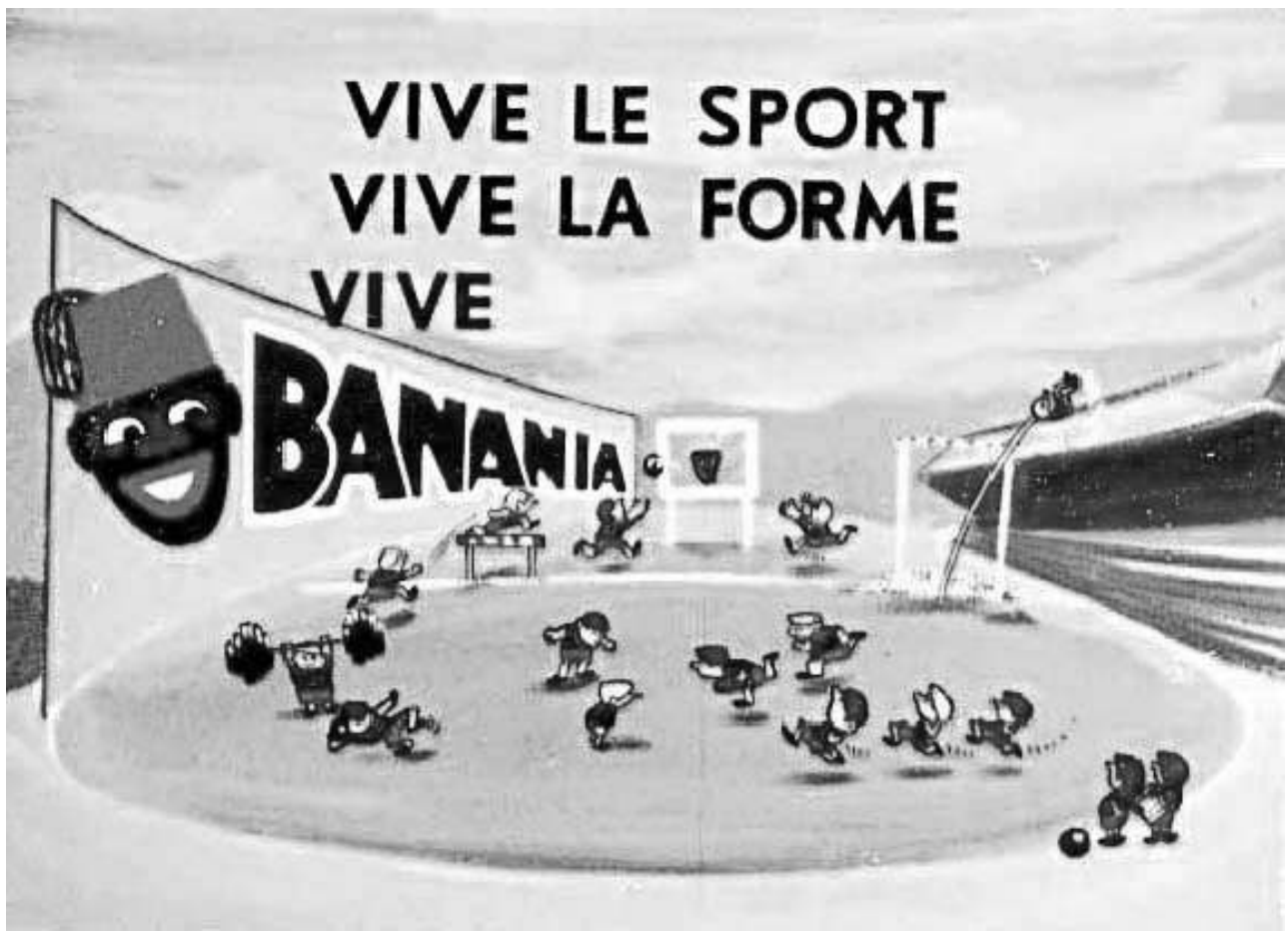

Figure 7 : amorce publicitaire, film le Hand-ball, 1960-1965

28 L'étude des films fixes nous conduit à traiter en parallèle deux dimensions fortement mêlées. L'image est incluse dans un récit, elle est l'occasion, le motif, le but, l'illustration du discours ; mais dans le même temps elle porte des intentions (pas toujours celles du pédagogue), elle constitue le discours lui-même. Il y a dans chaque image pédagogique le double jeu de la dénotation et de la connotation. L'image prend forme dans et par le 
discours et dans le même temps elle induit la forme du discours. Cette double détente enracine la fascination du pédagogue pour l'image et fait naître la méfiance qu'elle lui inspire. L'enjeu symbolique du discours est posé : qui maitrise le discours? L'enseignant ou l'image? Si l'on pose, comme point de vue théorique mais aussi professionnel, l'enjeu symbolique de l'artefact, on pose la maitrise a priori de l'instrument que porte l'objet. L'enjeu symbolique peut alors être promu (ou non) par le maitre dans l'espace interpsychique de la situation d'enseignement. Utiliser des outils de communication nécessite qu'aux compétences scolaires s'ajoutent des compétences d'utilisateur. Tous les ingrédients sont présents pour créer une méfiance vis-à-vis de l'analogon technologique.

Elle est quelquefois justifiée si l'on se reporte aux exemples que l'on trouve dans certaines vues de notre fonds. Nous trouvons par exemple des vues qui sont peu lisibles. Non qu'elles soient floues ou mal cadrées, mais parce qu'elles n'illustrent pas très bien le contenu qu'elles sont censées présenter. Malgré l'ajout de flèches ou de commentaires, on ne perçoit pas la portée pédagogique de l'image.

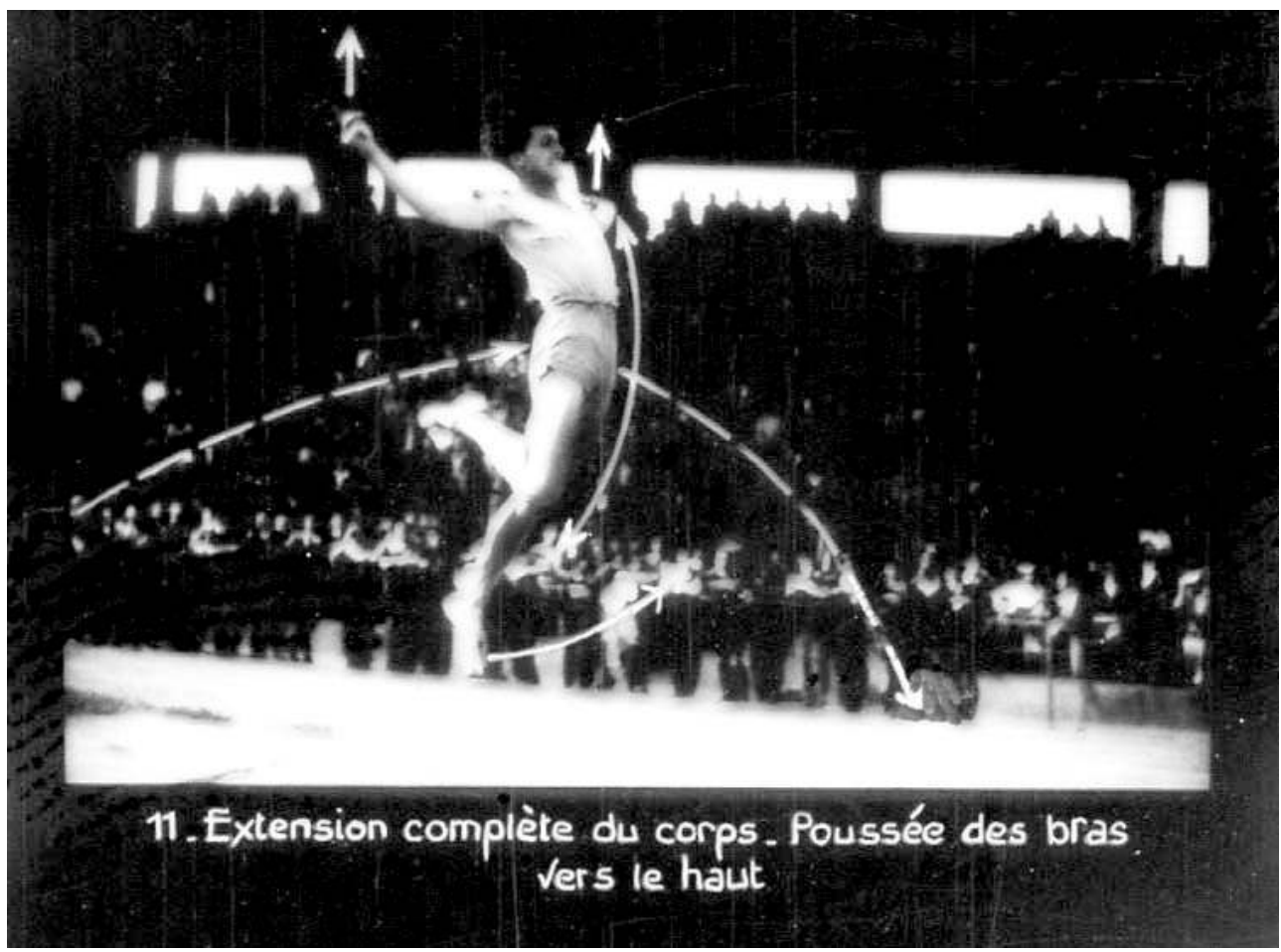

Figure 8 : film le saut en longueur, 1950

D'autres sont contradictoires avec le texte de la légende : dans l'image qui suit on lit en préambule « le javelot est tenu dans la main gauche ». 


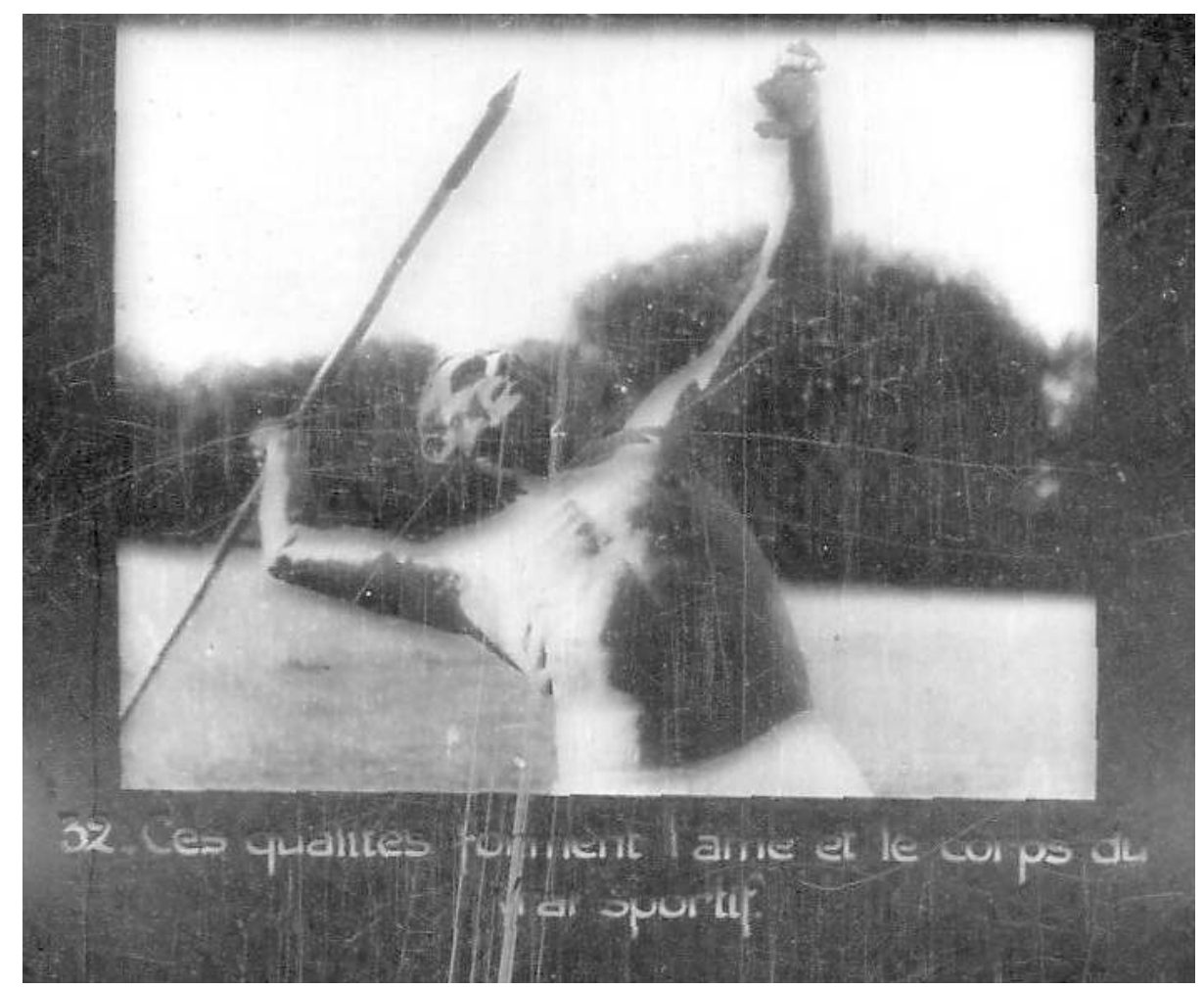

Figure 9 : vue du film sport et santé, 1935 - 1940

31 D'autres enfin représentent, surtout lorsqu'il s'agit de dessins, des aberrations techniques.

Autant de bonnes raisons qui confortent le pédagogue dans son désir de maîtrise et dans sa crainte d'être trahi par l'image. Le pédagogue se méfie de l'image analogique comme icône trompeuse.

\section{L'image et le discours pédagogique : la métaphore}

Prendre du recul, en pédagogie comme ailleurs, consiste à étudier le discours des acteurs. Il faut entendre par discours tout ce qui concourt à échanger une pensée dans un procès communicationnel. Le discours tient ensemble deux fondements de l'acte pédagogique : l'exigence de vérité et la précision de la communication. Le discours s'appuie alors sur une rhétorique constitutive de la pensée éducative elle-même. On retrouve cette rhétorique dans la combinaison des langages que le pédagogue utilise pour arriver à ses fins (la parole, l'image, l'expérience, l'exemple...). Toutes les «figures» du discours, verbal et non verbal, sont utilisées pour exprimer et développer la pensée, pour « faire passer le message ». La métaphore est donc, elle aussi, convoquée à cette affaire.

34 L'image pédagogique mêle la visée didactique de transmission des connaissances et la dimension axiologique de l'École. C'est pour cela que la parole pédagogique est consommatrice de métaphores. En déplaçant le contenu (le signifié ou l'interprétant peircien: ce que signifie l'image) sur un autre objet on le décale de son contexte (le référent : ce que représente l'image, le réel) ce qui crée l'espace suffisant pour en montrer la charge axiologique (le signifiant ou le representamen peircien : ce que l'on perçoit de l'image). Là encore, la création de l'espace et ce qui l'habite sont potentiellement des 
instruments psychologiques à partager dans l'espace inter-psychique de la situation d'enseignement.

Nadine Charbonnel pose de façon très précise la difficulté à chercher dans notre corpus les éventuelles métaphores. Nous serons toujours sous le coup de l'interprétation, nous l'acceptons lorsque nous passons les films fixes au crible de la recherche métaphorique. Cette recherche nous a conduits à différencier ce qui ressort des vues à lecture directe qui représentent l'objet sans autre intention que d'en donner une image, et les vues que l'on peut qualifier de métaphores. Ces dernières, montrant le fait sportif, peuvent signifier autre chose, elles recouvrent un autre signifié. La grande difficulté est de juger de l'aspect volontaire ou involontaire de la métaphore. Rechercher, systématiquement, l'instrument psychologique représente une contrainte nécessaire pour dépasser une réception trop rapide de l'artefact qui, certaines fois, frise la naïveté.

Certaines images sont volontairement métaphoriques et cela sans nul doute possible. Nous trouvons par exemple la représentation d'un ciseau, l'outil, superposée à un ciseau, un battement de jambes en natation. Dans ce cas, la métaphore est claire, c'est une comparaison imagée, un instrument psychologique généralisable, visant à renforcer l'instrument technique moteur qui est le battement de jambes. Il y a alors une extension $\mathrm{du}$ sens de l'image, au mot et du mot à la conscience (en l'occurrence une technique de nage). Le sens de la métaphore est celui qui va du geste sportif à un référent autre mais qui est utilisé pour ses propriétés communes. Le signifié de l'image est alors renforcé par la comparaison, enfin le croit-on. À la suite de N. Charbonnel, nous les qualifions de métaphores vives tant la signification est appuyée et l'effet recherché évident.

Dans d'autres cas le signifié est caché et seule l'analyse le découvrira. La métaphore est cette fois insidieuse voire perfide, elle vise à enseigner autre chose que le référent. La métaphore est alors endormie. Elle n'attend que le déclic qui la révèlera alors que le lecteur l'aura déjà parcourue à plusieurs reprises sans qu'elle lui apparaisse. Dans un film présentant les Jeux Olympiques de 1936, nous pouvons relever, dans nombre de vues, des métaphores guerrières. Une vue montre un arbitre, dont on ne doute pas qu'il est choisi pour son allure sévère. Il prépare son matériel devant une batterie d'engins de mesure. Il pointe son pistolet non vers le haut comme tout starter le ferait mais dans la direction d'un hypothétique quidam. Dans ces vues il y a un changement du sens de l'image : on croit voir du sport et on voit de la propagande. Le sens de la métaphore est inversé et c'est le geste sportif qui est métaphorisé par une intention qui n'est peut-être pas que didactique. Avec le recul de l'histoire on peut interpréter le contenu informel de cette image comme l'intrusion d'un signifié qui n'a rien à voir avec le projet scolaire de présenter les Jeux Olympiques. 


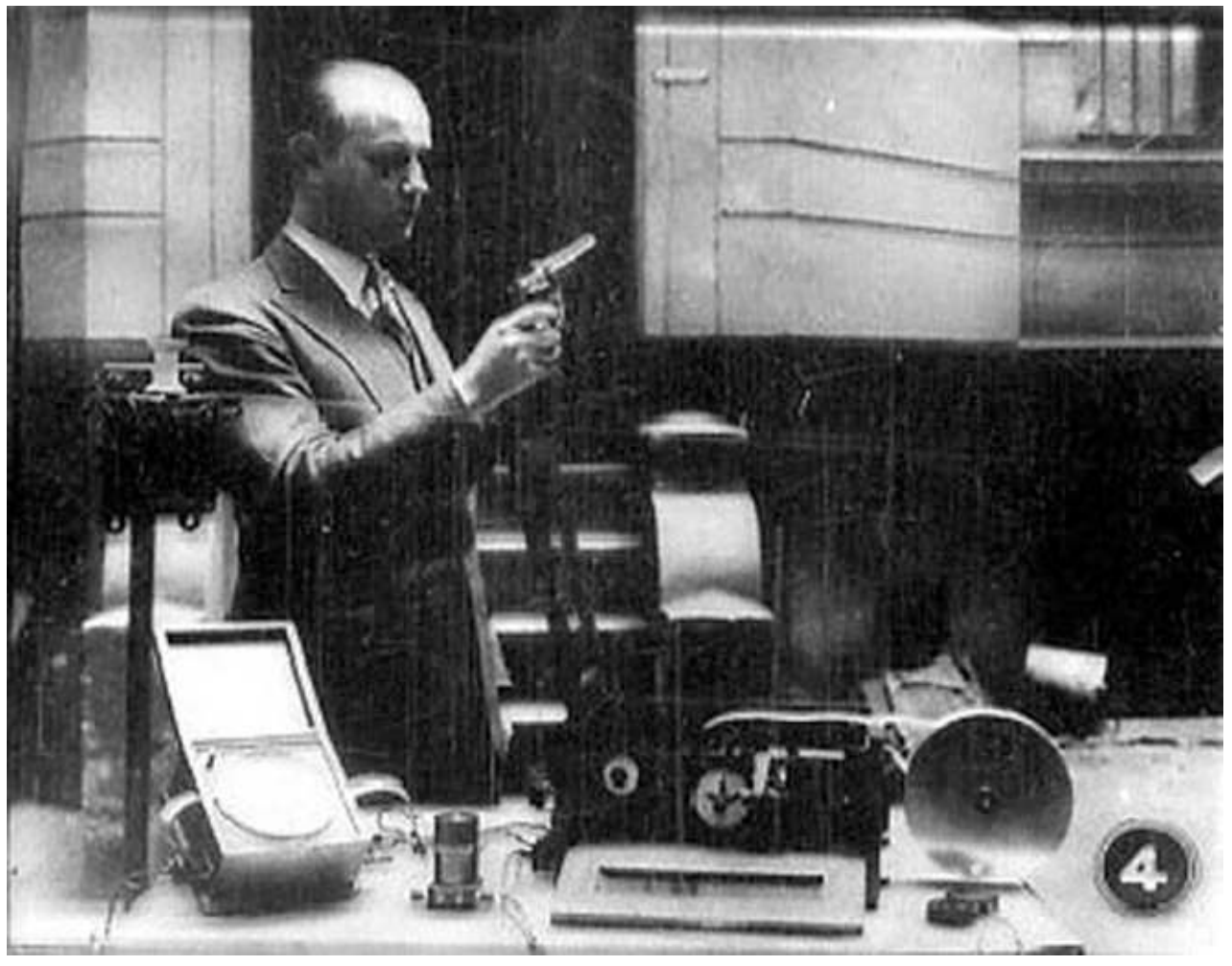

Figure 10 : vue du film les jeux Olympique de Berlin, 1936-1938 métaphores que l'on retrouve sont courants dans le discours de l'éducation physique : la force, le courage, l'abnégation, le combat, la discipline, la rigueur, l'effort, la nature, l'entraide, etc. Dans ce cas, le signifié est tellement habituel, culturellement assimilé, que la métaphore perd de son impact et meurt de sa belle mort.

D'autres images enfin, tendent plus vers l'allégorie que vers la métaphore. Les vues utilisent les symboles classiques de l'élévation, de l'aérien, de la grâce, de la pureté, etc. 


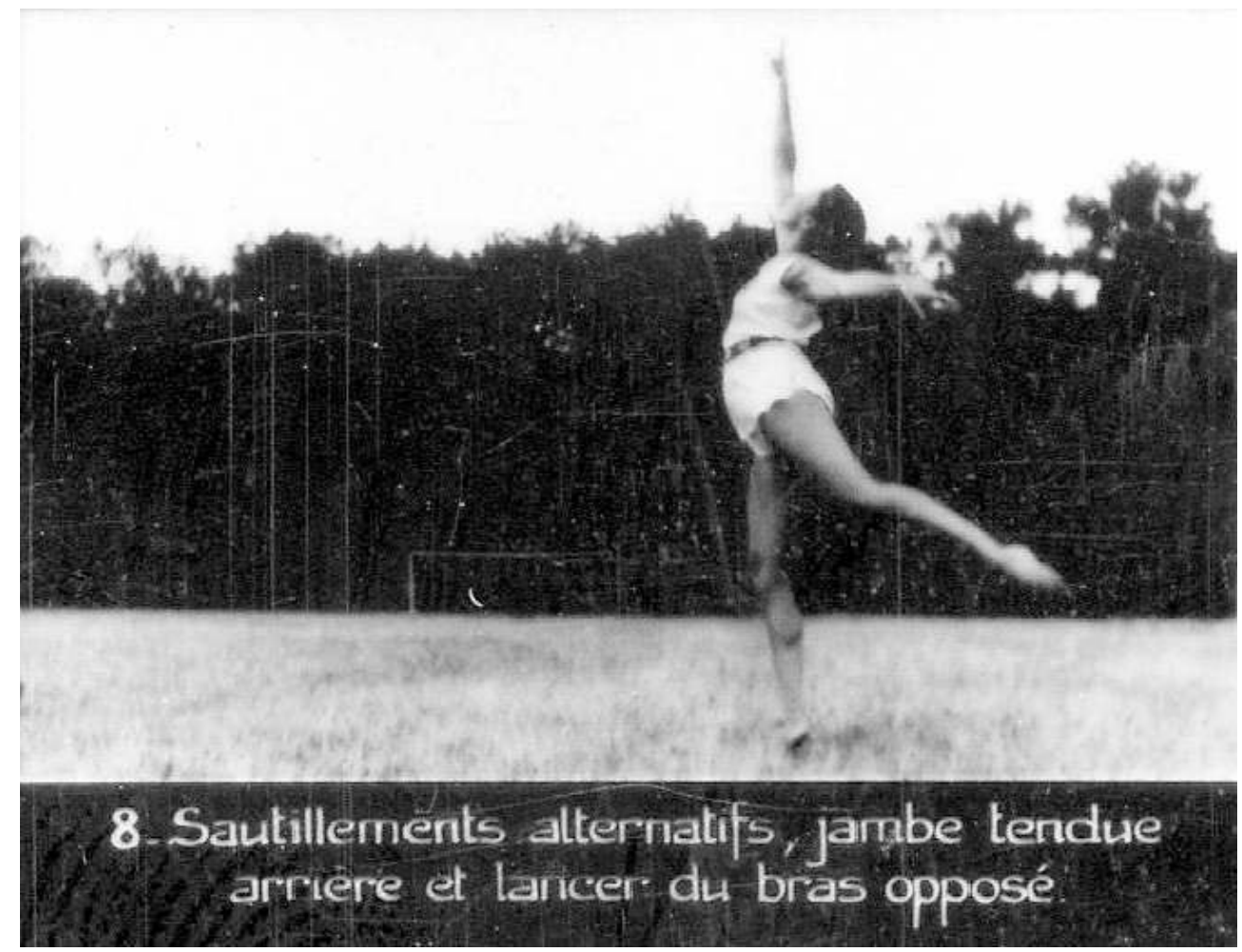

Figure 11 : vue du film Gymnastique rythmique II, 1930-1935

Dans ces vues le symbole est le signifiant d'un geste sportif dont le signifié se trouve renvoyé au rang de faire valoir. Ce primat de l'idée, du symbole, sur le référent définit pour nous la différence entre métaphore et allégorie. Ce sont deux figures qui du point de vue de l'importance du signifié sont diamétralement opposées.

\section{Conclusion}

41 La photographie parle d'elle-même et l'on peut parler de la photographie. On voit alors l'articulation des deux dimensions de notre travail: une sémiotique de l'image et une sémantique du discours pédagogique. Ce qui déroute le pédagogue c'est qu'il peut être dépassé par le langage photographique qui pourrait pervertir sa propre parole. C'est peut-être là qu'il faut situer la méfiance du pédagogue vis-à-vis de l'image. Mais c'est aussi dans la parole que le pédagogue ancre son action. D'où le rapport ambigu qu'il entretient avec l'image : il s'en méfie mais a recours à elle systématiquement. Il se méfie de l'icône trompeuse mais se sert de la métaphore.

42 La métaphore est un procédé d'expression, un moyen plus efficace de se faire comprendre, de dire de façon non directe. L'écueil est qu'un signifié caché peut, subrepticement, pervertir le projet même d'éduquer.

43 Pourquoi une image fait apprendre ? Notre hypothèse est instrumentale : une image n'est pas qu'une trace, elle porte un « instrument psychologique ». 
Publicité Filmostat, Manuel général de l'instruction primaire, journal hebdomadaire des instituteurs et institutrices $96 \mathrm{e}$ année, $\mathrm{N}^{\circ} 23,2$ février 1929, p. 72.

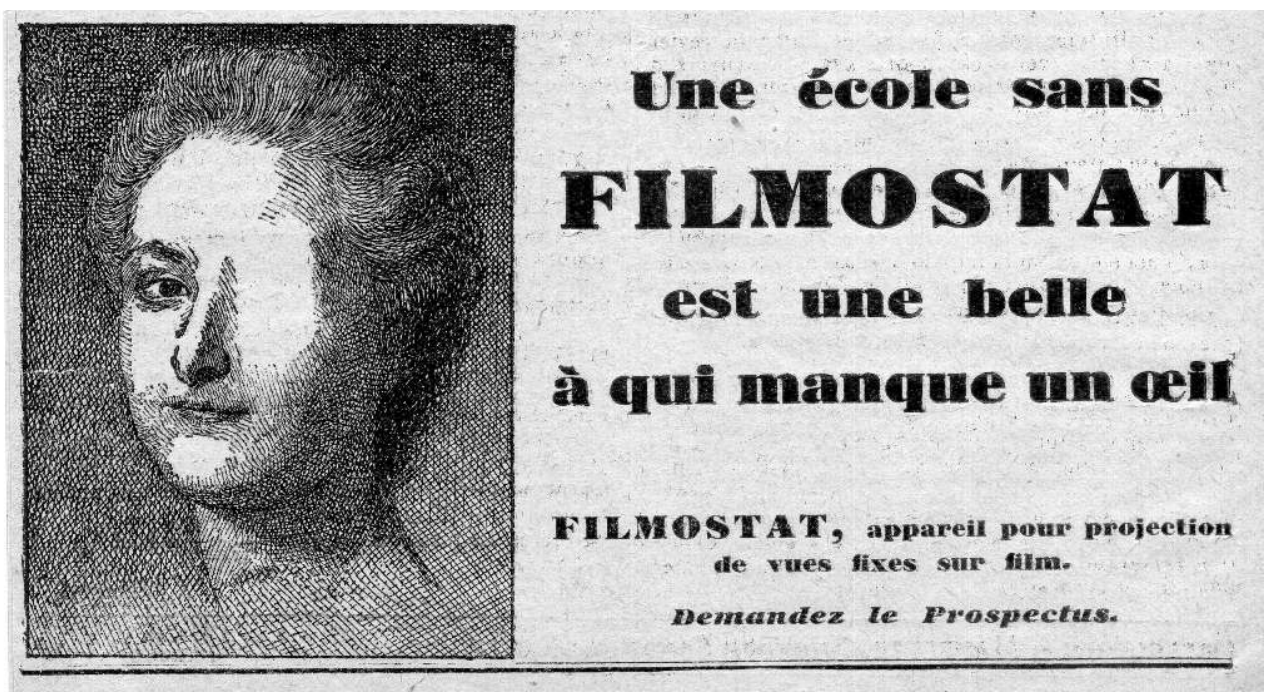

\section{BIBLIOGRAPHY}

Barthes, R. (1964). Éléments de sémiologie. Communication 4, Recherches Sémiologiques, École Pratique des Hautes Études-Centre d'études des Communication de Masse. Paris : éditions du Seuil.

Caumeil, J.-G. (2000). Contribution à une épistémologie de l'éducation physique et sportive scolaire. Du savoir de l'activité motrice aux éléments d'une pédagogie du sens. Tome 1. Lille : Presses universitaires du Septentrion, coll. Thèse à la carte.

Caumeil, J.-G. (Dir.) (2006). Fonder les savoirs scolaires. Épistémo-anthropologie et médiation pour un regard nouveau sur l'éducation. Lyon : SCERÉN et IUFM de l'académie de Lyon, coll. Savoirs en pratiques.

Caumeil, J.-G., Charroin, P., Lignon, F., Nourrisson, D. (2010). Fixité de l'image, mobilité des corps. L'enseignement des activités physiques et sportives par le film fixe. Clermont-Ferrand : Impr. Diazo, 200 p. + CD Rom.

Davidov, V.-V. (1972). Les types de généralisation au cours de l'apprentissage. Moscou : Pédagoguika (texte non traduit).

Debray, R. (1992). Vie et mort de l'image. Une histoire du regard en Occident. Paris : Gallimard, Coll. Bibliothèque des Idées.

Gagnepain, J. (1993). Du vouloir dire. Traité d'épistémologie des sciences humaines. Bruxelles : De Boeck Université, coll. Raisonnance, 3 vol.

Herskovits, M.-J. (1950-1967). Les bases de l'anthropologie culturelle. Paris : François Maspero Éditeur, Coll. Petite collection Maspero. 
Kozulin, A. (Dir.) et al. (2009). Vygotski et l'éducation. Paris : Retz.

Lamotte, J.-L. (2001). Introduction à la théorie de la médiation. L'anthropologie de Jean Gagnepain. Bruxelles : De Boeck Université, coll. Raisonnances.

Leontiev, A.-N. (1976). Le développement du psychisme. Paris : Éditions Sociales.

Martin, M. (1982), Sémiologie de l'image et pédagogie : pour une pédagogie de la recherche.

Paris : Presses Universitaires de France.

Rivière, A. (1984). La psicología de Vygotsky. Revue Infancia y Aprendizaje 27/28, 32-40.

Schneuwly, B., et Bronckart, J.-P., (Dir.) (1985). Vygotsky aujourd'hui. Neuchâtel (Switzerland)-

Paris : Delachaux et Niestlé, coll. Textes de Base en psychologie.

Vygotski, L.-S. (1983). Histoire du développement des fonctions psychiques supérieure. Euvres choisies en 6 volumes, volume 3. Moscou : Ed Matuskin, A.M., coll. Pédagogie.

Vygotski, L.-S. (1962). Thought and language. Cambridge : MIT Press.

Wartofski, M.-W. (1979). Models : Representation and scientific understanding. Dordrecht, Holland. Boston : D. Reidel.

\section{NOTES}

1. L'orthographe « artefact » est volontaire. Elle signifie que nous faisons référence au concept de la théorie instrumentale et non pas du terme français « artéfact » qui renvoie à tout autre chose. 2. Le fonds, de plus de 10000 films actuellement, est donc à disposition des chercheurs.

3. Cette nouvelle forme d'enseignement s'appuyait, par ailleurs, sur les pratiques innovantes développées par les courants de l'Éducation Populaire qui les ont expérimentées très tôt auprès des publics issus des vagues d'immigrations dues à l'exploitation du charbon sur tout le territoire national.

4. Barthes, R. (1964). Éléments de sémiologie. Communication 4, Recherches Sémiologiques, École Pratique des Hautes Études-Centre d'études des Communication de Masse. Paris : éditions du Seuil, 91-136, p. 92.

5. Barthes (lire par exemple Le bruissement de la langue; Essais critiques, "L'Analyse rhétorique ", IV), définit le signe comme la clé permettant de saisir la signification de l'univers qui est un grimoire peuplé d'indices. Tout message comprend au moins un plan d'expression (le signifiant) et un plan de contenu (le signifié). La jonction de ces deux plans forme le signe. Pour le moment nous fonctionnons à partir de cette approche qui, en l'état d'avancement de nos recherches, est suffisante. Cependant, nous avons conscience qu'elle est probablement simpliste pour le spécialiste.

6. Martin, M. (1982). Sémiologie de l'image et pédagogie : pour une pédagogie de la recherche. Paris : Presses Universitaires de France, $\mathrm{p}$ 17. «La sémiologie de l'image établit que toute lecture iconique passe par un code à caractère spécifique. Nous insistons sur le phénomène de codification, qui, même figuratif, n'est certes pas innocent (en italique dans le texte). Ce n'est pas parce que le lecteur a l'impression de naturalité que celle-ci existe effectivement. En outre, l'image supporte facilement diverses connotations qui jouent un rôle essentiel au niveau de la signification ».

7. Cité par Roland Barthes, op. cit. p. 94. Hjelmslev, L. (1959). Essais linguistiques. Copenhague : Nordisk- Sprog-og Kulturforlag, p 69. 
8. Martin, M. (1982). Sémiologie de l'image et pédagogie. Pour une pédagogie de la recherche. Paris: Presses Universitaires de France, coll. Pédagogie d'aujourd'hui, dirigée par Gaston Mialaret, p. 24-25.

9. Sachant que, dès la fin du XIXe siècle, Ferdinand Buisson développe une politique très volontariste à l'égard des classes sociales défavorisées, les ouvriers et les immigrants, enfants et adultes, qui s'appuie sur la projection de vues sur plaques de verre. Il fait de la projection de vues éducatives le fondement de la pédagogie nouvelle.

\#Notes

INDEX

Mots-clés: artefact, image, théorie instrumentale, Vygotski

Keywords: artifact, instrumental theory, Vygotsky

\section{AUTHOR}

\section{JEAN-GUY CAUMEIL}

Maître de Conférences, STAPS, ESPE Lyon1, CRIS DPO université Lyon 1, EA 647, EFEA 